\title{
CESE Report \\ A Case of Retrieval of a Migrated Coil with a Stent Retriever
}

Norio Fujii, Takayuki Iwakami, and Jaehyun Son

Objective: Coil migration is a complication related to endovascular coil embolization. In this study, we report a patient in whom an intraoperatively migrated coil was successfully retrieved using a stent retriever.

Case Presentation: A 57-year-old male. To treat an unruptured aneurysm of the right internal carotid artery, endovascular coil embolization was performed. During embolization, one end of a framing coil migrated into the parent artery, and it was retrieved using a microsnare. However, this resulted in the extra-aneurysmal deviation of a filling coil. We tried to retrieve it using a microsnare, but the formation of a coil mass made retrieval difficult. Eventually, retrieval with a stent retriever was successful. After operation, mild subarachnoid hemorrhage and slight muscle weakness of the left upper/ lower limbs were observed, but recovery was achieved during the course, and the patient was discharged.

Conclusion: To our knowledge, no study has reported retrieval with a stent retriever for the management of coil migration in Japan. However, this method was considered to be effective. We report this patient, and review the literature.

Keywords $>$ endovascular coil embolization, unruptured cerebral aneurysm, coil migration, stent retriever

\section{Introduction}

The therapeutic effects and safety of endovascular coil embolization for cerebral aneurysms have improved with advances in devices/coils. This procedure will be increasingly selected in the future. However, the risk of serious sequelae is still present, and the incidence of serious endovascular-treatment-related complications (modified Rankin Scale [mRS] score: $\geq 3$, Mini-Mental State Examination [MMSE] score: $<24$ ) is $7.3 \%$ according to the International Study of Unruptured Intracranial Aneurysms (ISUIA), with a mortality rate of $1.8 \% .^{1)}$ As complications during coil embolization, thromboembolism, and intraoperative aneurysmal rupture are

$\overline{\text { Department of Neurosurgery, Tomishiro Central Hospital, Tomigu- }}$ suku, Okinawa, Japan

Received: June 7, 2017; Accepted: September 19, 2017

Corresponding author: Norio Fujii. Department of Neurosurgery, Tomishiro Central Hospital, 25 Ueda, Tomigusuku, Okinawa 901-0243, Japan

Email: fujii-nori@hotmail.com

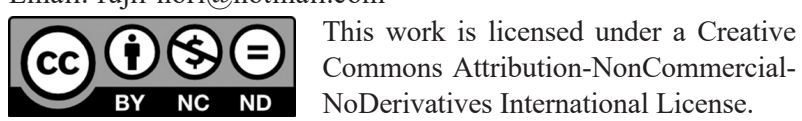

(C)2018 The Japanese Society for Neuroendovascular Therapy commonly observed, ${ }^{2)}$ but coil migration is also a non-negligible complication that may occur during operation. The incidence of coil migration is reportedly $2 \%-6 \%,{ }^{3-7)}$ and extensive cerebral infarction may induce serious sequelae; therefore, prompt management is necessary if coil migration occurs. In this study, we report a patient in whom coil migration developed twice during operation, but the two coils were successfully retrieved using a microsnare and stent retriever, and review the literature.

\section{Case Presentation}

Case: A 57-year-old male.

Complaint: Not contributory.

Medical history: In May 2016, mitral valve plasty for mitral valve regurgitation was performed.

Present illness: MRI prior to open heart surgery revealed an unruptured cerebral aneurysm, and the patient was referred to our department. Endovascular coil embolization with a balloon-assisted technique was scheduled.

Physical examination: Consciousness was clear. There was no neurologic deficit.

Radiological findings: Cerebral angiography showed an aneurysm on the ventral side of the right internal carotid 
artery (C3). Its size was $4.2 \times 3.8 \times 5 \mathrm{~mm}$, with a neck measuring $2.8 \mathrm{~mm}$ (Fig. 1).

Cerebral endovascular treatment: The oral administration of aspirin at $100 \mathrm{mg} /$ day and clopidogrel at $75 \mathrm{mg}$ /day was started 7 days before embolization. Under general anesthesia, a 7.2 Fr sheath was inserted into the right femoral artery, and heparin at 5000 units was systemically administered, with an activated clotting time (ACT) of 321 seconds. After inserting a 7 Fr FUBUKI hard $90 \mathrm{~cm}$ (Asahi Intecc Co., Ltd, Aichi, Japan) into the right internal carotid artery, a Scepter XC $4 \times 11 \mathrm{~mm}$ (MicroVention TERUMO, Tustin, CA, USA) was guided to the aneurysmal neck. Subsequently, a Headway 17 pre-shaped angle 90 degrees microcatheter (MicroVention TERUMO) was guided into the aneurysm using a NEUROUTE 14 microguidewire (MEDICO'S Hirata, Osaka, Japan). An Axium prime 3D $4 \times 100 \mathrm{~mm}$ and Axium prime helix $3 \times 40 \mathrm{~mm}$ (Medtronic, Minneapolis, MN, USA) were inserted into the aneurysm, but a portion of the coil mass protruded into the parent artery while filling an Axium prime helix $2.5 \times 30 \mathrm{~mm}$ (Fig. 2A and 2B). The Scepter $\mathrm{XC}$ was inflated so that the protruding coil might return into the aneurysm. However, a portion of the Axium prime 3D $4 \times 100 \mathrm{~mm}$ strayed into the parent artery, migrating to the horizontal segment (M1) of the right middle cerebral artery via blood flow (Fig. 2C). Using a Goose neck snare $4 \mathrm{~mm}$ (Medtronic), the coil end that had migrated was captured (Fig. 3A), but coil unraveling occurred in the process of retrieval, making retrieval impossible (Fig. 3B). The capturing operation was relieved, and the microsnare was removed. Then, the coil migrated to a distal area via blood flow, forming a coil mass (Fig. 3C). The tip of the delivery wire CHIKAI 14 (Asahi Intecc Co., Ltd) of the Scepter XC was captured with the Goose neck snare $4 \mathrm{~mm}$, and the mass-forming coil was surrounded/retrieved by simultaneously pulling the microsnare and CHIKAI 14 (Fig. 3D). Although retrieval was successful, the Axium prime helix $3 \times 40 \mathrm{~mm}$ deviated to the extra-aneurysmal area, migrating to the right $\mathrm{M} 1$ area, with mass formation. As retrieval with a microsnare was considered difficult, a Trevo pro 18 microcatheter (Stryker Neurovascular, Kalamazoo, MI, USA) was guided into the superior trunk (M2) of the right middle cerebral artery. At this point, the deviating coil had further migrated to an area adjacent to the bifurcation of the right middle cerebral artery (Fig. 4A). A Trevo XP ProVue Retriever $6 \times 25 \mathrm{~mm}$ (Stryker Neurovascular) was guided/developed. And a light counter pressure was given

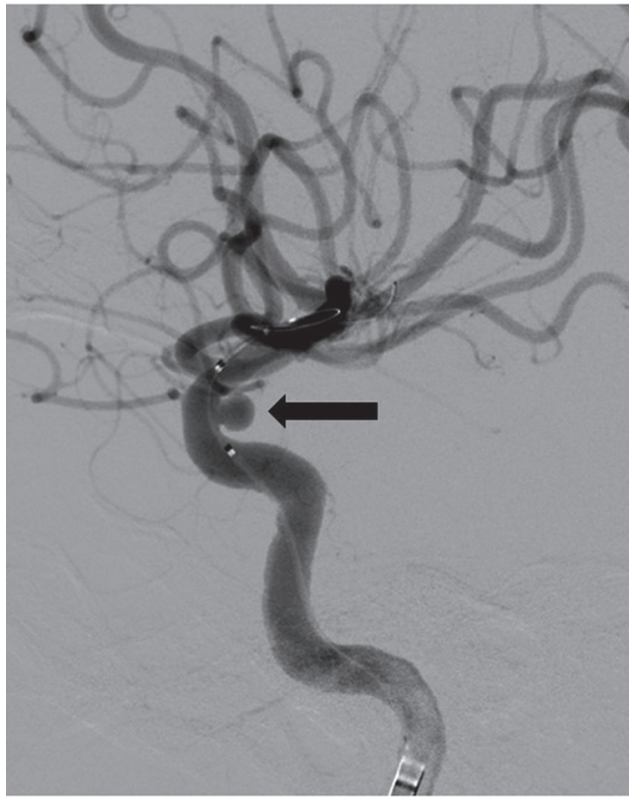

Fig. 1 Right internal carotid arteriogram shows unruptured internal carotid aneurysm (arrow).

so that the coil mass might be entwined with a stent strut (Fig. 4B). Subsequently, the delivery wire of the stent retriever was carefully pulled, and the deviating coil was retrieved. It was confirmed that the two deviating coils had been retrieved. A 7 Fr FUBUKI hard $90 \mathrm{~cm}$ was again inserted into the right internal carotid artery, and intracranial angiography was performed. There was no vascular occlusion or extravasation; therefore, coil embolization was resumed. After guiding a Scepter XC $4 \times 11 \mathrm{~mm}$ to the aneurysmal neck, a Headway 17 pre-shaped angle 90 degrees microcatheter was guided into the aneurysm. An Orbit GALAXY Complex fill $4 \times 100 \mathrm{~mm}$ (Johnson \& Johnson, Fremont, CA, USA), Axium prime helix $2.5 \times$ $30 \mathrm{~mm}$, Axium prime helix $1.5 \times 20 \mathrm{~mm}$, Axium prime 3D $1.5 \times 20 \mathrm{~mm}$, Axium prime 3D $1 \times 20 \mathrm{~mm}$, and Target helical nano $1 \times 20 \mathrm{~mm}$ (Stryker Neurovascular) were inserted, and complete embolization of the aneurysm was confirmed (Fig. 5). Postoperative brain CT revealed thin subarachnoid hemorrhage of the right sylvian fissure (Fig. 6), and respiratory care with an artificial respirator was conducted under sedative therapy on the day of operation.

Post-treatment course: On brain CT the day after operation, there was no exacerbation of hemorrhage. On awakening, slight muscle weakness of the left upper limb (Manual Muscle Test [MMT]: 4) was noted, but recovery was achieved during the course. The patient was discharged 8 days after operation. 


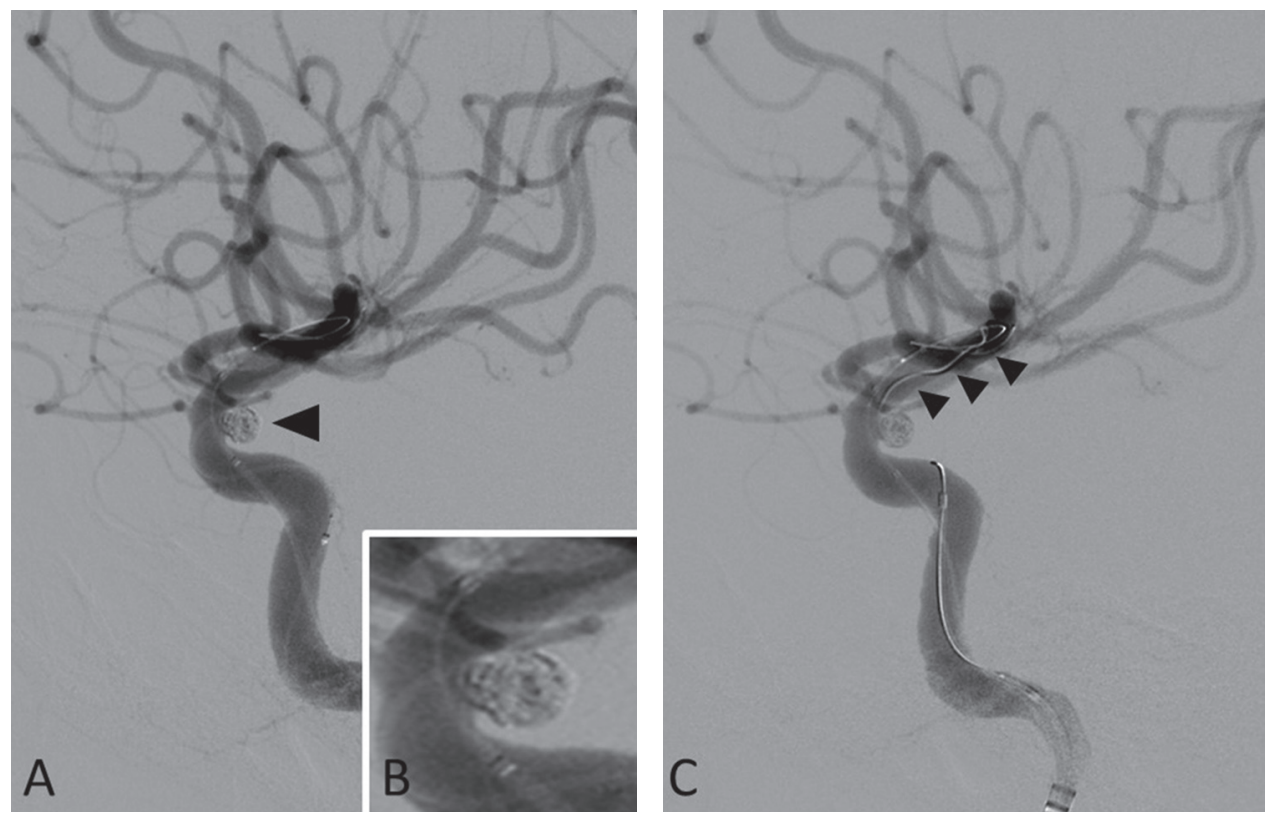

Fig. 2 (A) Right internal carotid arteriogram shows protrusion of coil (arrowhead) toward right internal carotid artery during the procedure. (B) Enlarged view of right internal carotid artery around the aneurysm. (C) Right internal carotid arteriogram shows migration of a part of coil (arrowheads) during the procedure.

\section{Discussion}

The results and safety of endovascular treatment for cerebral aneurysms have improved with advances in devices/ coils. This procedure will be increasingly applied in the future. However, the risk of serious surgery-associated complications or death is still present. According to the ISUIA, the incidence of serious endovascular-treatmentrelated complications is $7.3 \%$, and the mortality rate of $1.8 \%{ }^{1)}$ A study in Japan indicated that the incidence of endovascular-treatment-related complications was $9.1 \%$, and that the mortality rate was $0.31 \%{ }^{8}{ }^{8}$ Primary intraoperative complications include thromboembolism and aneurysmal rupture. The incidences of thromboembolism and intraoperative rupture of unruptured aneurysms are $2.5 \%-28 \%$ and $0 \%-2.4 \%$, respectively. ${ }^{2,9,10)}$ However, other unexpected intraoperative complications have also been reported with an increase in the frequency of performing endovascular coil embolization. These include coil migration, and its incidence during endovascular coil embolization is $2 \%-6 \% .^{3-7)}$ Casasco et al. reported that the coil-migration-related mortality rate was $2.8 \%$, and that the moderate neurologic morbidity rate was $2.8 \%{ }^{11)}$; prompt decision-making is required for specialists in endovascular treatment. Factors involved in coil migration include wide-neck aneurysms with a dome/neck ratio of $<2$, unstable microcatheter positioning, unstable intraaneurysmal embolization related to the use of undersized/ long coils, tortuous blood vessels, and areas with a high flow velocity. ${ }^{6,7,12)}$ Under such circumstances, caution is particularly needed. A method to retrieve a deviating coil has not been established. However, microsnares, microbaskets, or tip-shape-modified microguidewires have been used as capturing devices, or Alligator retrieval devices/ Merci retrievers for mechanical thrombectomy or stent retrievers (only a few reports) have been adopted.4-7,12,13) In addition, some studies reported fixing a deviating coil to the vascular wall using a neck bridge stent or extirpation under craniotomy. However, these procedures have limitations: the long-term oral administration of antiplatelet drugs is required, and the procedures are highly invasive. Therefore, they are selected in many cases when retrieval with the above devices is difficult. ${ }^{14,15)}$ If a deviating coil migrates to an area where blood supply from leptomeningeal anastomoses may exist, follow-up by antiplatelet therapy may become a treatment option.

In the present case, a filling coil also migrated, following a framing coil. On the first deviation of the framing coil, coil unraveling occurred during retrieval with a snare, making direct coil retrieval impossible. Therefore, 

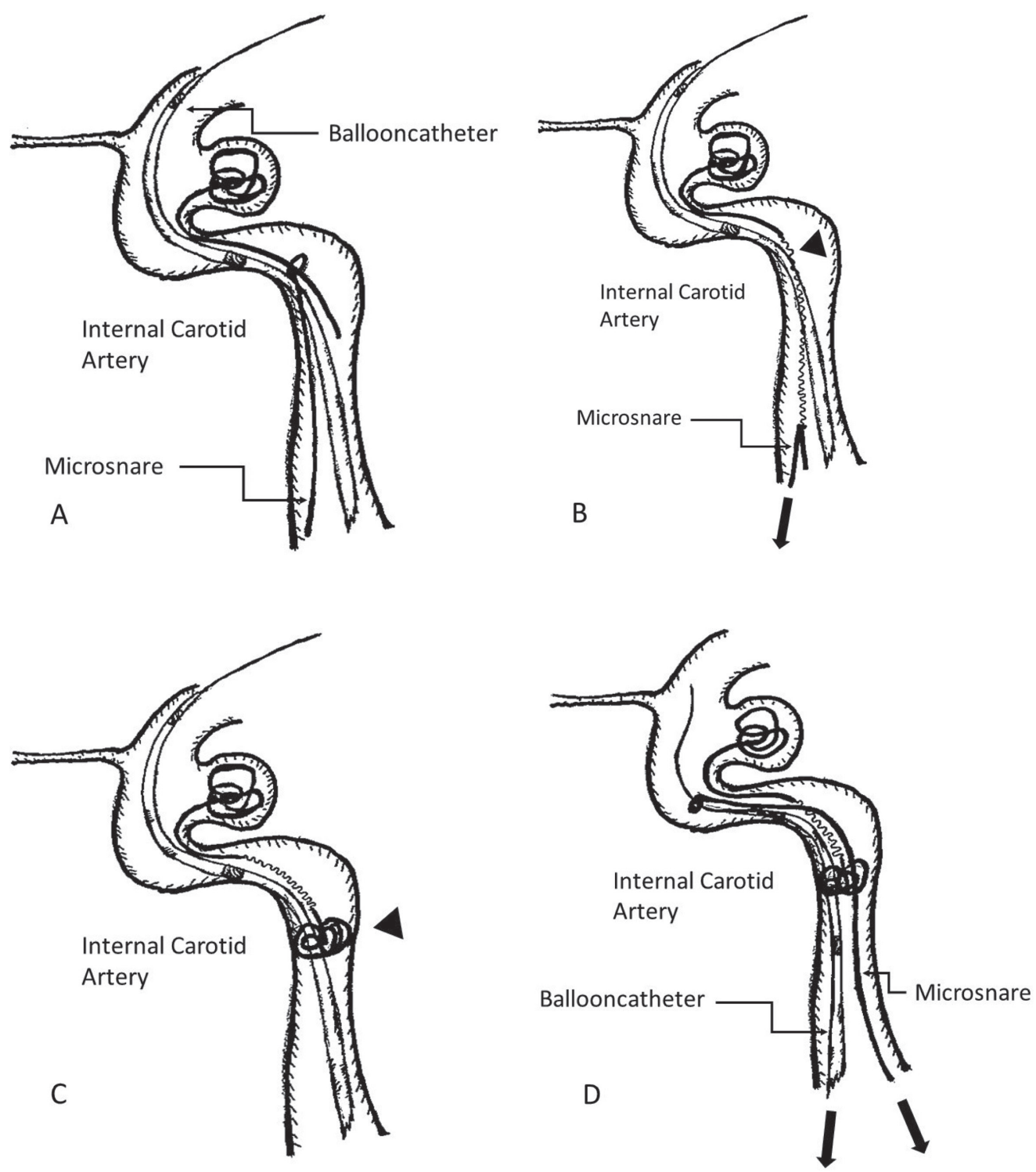

Fig. 3 Schematic of the retrieval method. (A) The microsnare was inserted and then captured the coil end. (B) The microsnare was pulled back and then a part of the coil was stretched (arrowhead). (C) The microsnare was retrieved with release of capturing the coil and then the stretched portion of the coil made a mass formation by the blood flow (arrowhead). (D) The microsnare was inserted again and captured the distal end of the delivery wire of the balloon catheter and then both of them were pulled back simultaneously (arrows).

we adopted a method to surround/retrieve the deviating coil with unraveling using the delivery wire of a balloon catheter and microsnare capturing it. However, with the impulse of the moment, the intra-aneurysmal filling coil with mass formation migrated to the horizontal segment (M1) of the middle cerebral artery. In the present case, a stent retriever was selected to retrieve the deviating filling coil for the following reasons: other foreign-body-retrieving devices, such as a microbasket-type foreign-body-removing device (Soutenir NV; Asahi Intecc Co., Ltd), were not available due to stock deficiency; and retrieval with a microsnare was considered difficult due to mass formation by the deviating coil. However, reflections obtained from our series led to the use of a stent retriever. Previously, we experienced coil migration during endovascular coil embolization for an unruptured aneurysm of the right internal carotid artery (C2). The coil migrated to the cortical branch of the right middle cerebral artery, and there was no problem regarding peripheral circulation; therefore, follow-up was considered, but we decided to fix the coil to the vascular wall using an Enterprise VRD (Johnson \& Johnson), considering the risk of thromboembolism. The postoperative course 

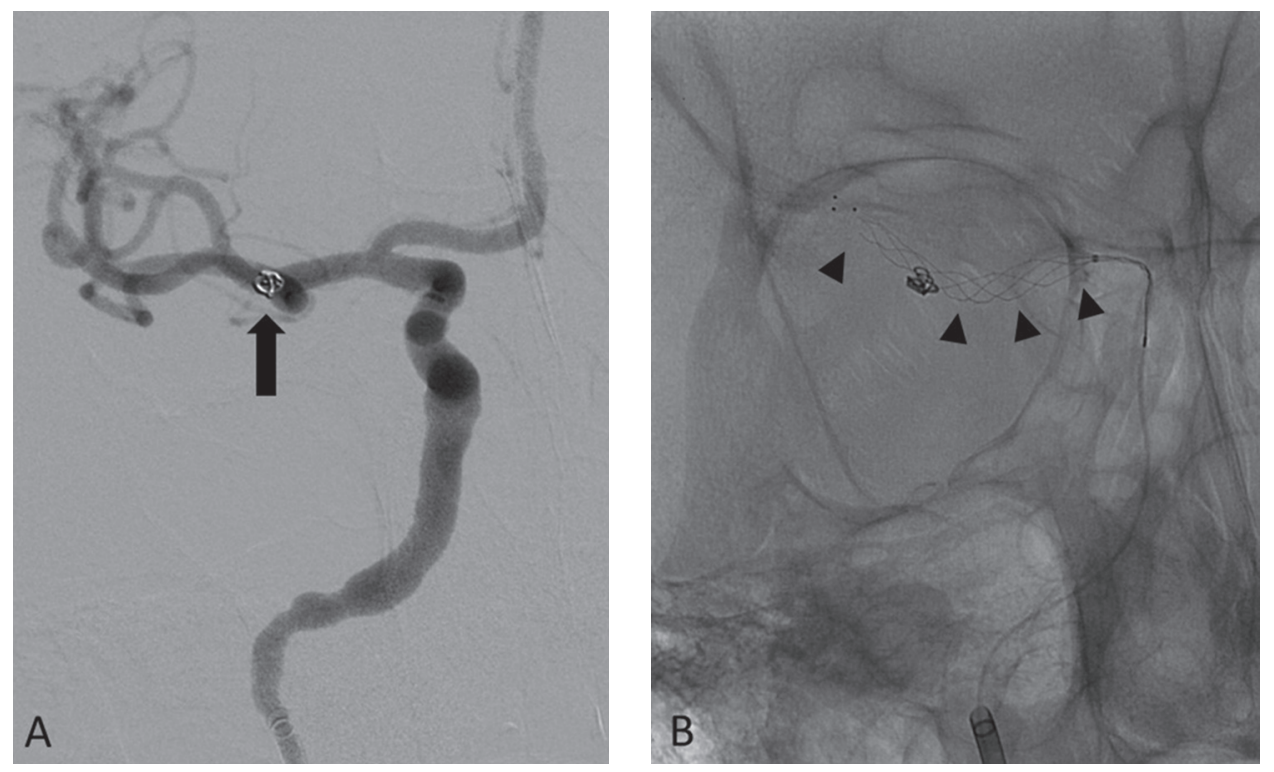

Fig. 4 (A) Right internal carotid arteriogram shows migration of the filling coil (arrow). (B) Right internal carotid arteriogram shows retrieval of the migrated coil with a stent retrieval device (arrowheads).

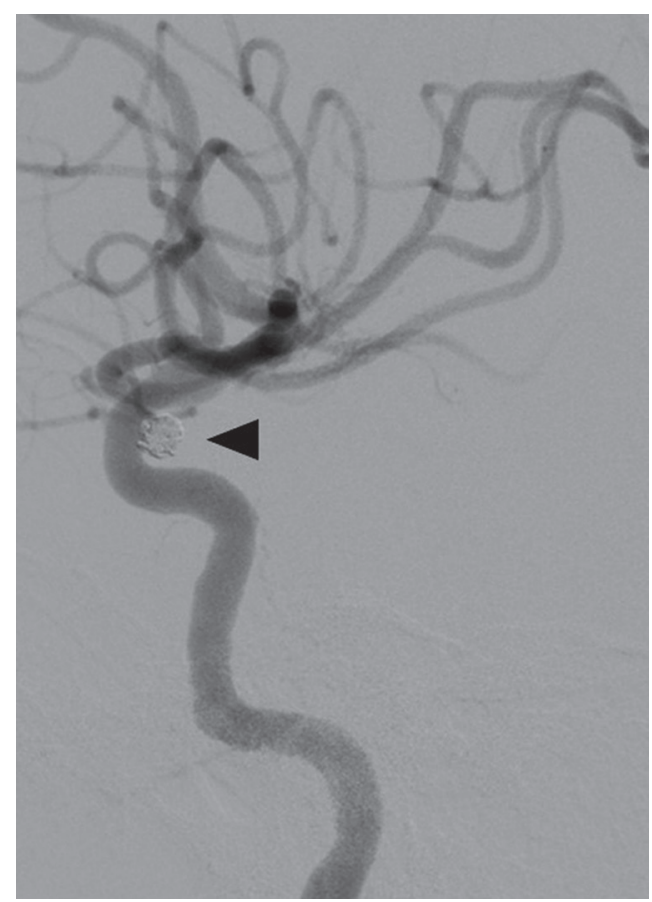

Fig. 5 Right internal carotid arteriogram shows complete occlusion of the unruptured internal carotid aneurysm in the coil embolization (arrowhead).

was favorable, and the patient was discharged. However, the long-term oral administration of an antiplatelet drug was necessary. Thereafter, we reviewed the literature, and found case reports in which coil retrieval with a stent retriever led to a favorable outcome ${ }^{7,16)}$; we have considered

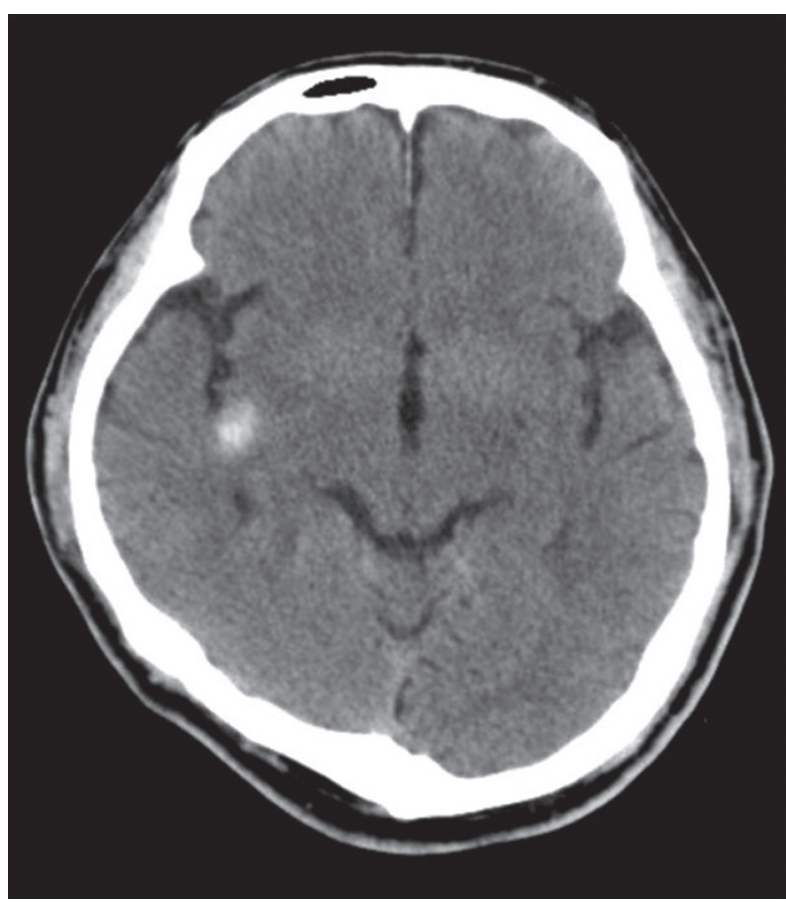

Fig. 6 CT scan after the coil embolization shows a thin subarachnoid hemorrhage in the right sylvian fissure.

this as a method to manage coil migration. For coil retrieval, a stent retriever has the following advantages: the size of a microcatheter can be selected in accordance with the vascular diameter, facilitating an approach to the periphery; and the elongation property of the device allows it to move along the vascular wall at the bifurcation 
or flexion point and capture a foreign body. As the merits of a Trevo XP ProVue, which was used in the present case, the shape of the distal end was modified from a tip shape to a flare shape, facilitating a more flexible structure against the vascular wall and reducing the risk of vascular injury during operations. In addition, this device is visualized as radiopacity, making it possible to evaluate the state of capturing at the vascular bifurcation or flexion point. However, there is no clinical evidence regarding its use beyond indication criteria. Furthermore, coil mass formation or distal coil migration may occur on lesion crossing. Based on these aspects, we consider that this device should be utilized as "a second option" for patients in whom retrieval with a microsnare or other retrieval devices is difficult, but not as a first option in the case of emergency.

In the present case, the device was guided so that a coil might be located in the active zone of a stent retriever, and developed while adding a light counter pressure on the delivery wire at the time of unsheathing. The coil was entwined with a stent strut. International studies presented technical notes for coil retrieval with a stent retriever. Nikoubashman et al. evaluated coil retrieval with a Trevo XP Provue in porcine blood vessels. In their experiment, standard thrombectomy or advanced coil-retrieval techniques (two groups) were performed after developing a stent, with a coil being in the active zone. In the latter, a deviating coil was surrounded/ retrieved by slightly pulling the stent on resheathing a microcatheter carefully. As a result, coil retrieval was successful in $11 \%$ of specimens in the standard thrombectomy group and $99 \%$ of specimens in the advanced coil-retrieval group. ${ }^{17)}$ To our knowledge, there were two patients in whom coil retrieval using a method similar to the advanced coil-retrieval technique was successful. ${ }^{16,18)}$ Further investigation may be necessary. However, when performing coil retrieval using a stent retriever, procedures, such as the advanced coilretrieval technique, should be considered.

In the present case, issues to be reflected on include device-size selection. We selected a device measuring $6 \mathrm{~mm}$ in diameter, as selected for standard thrombectomy, to capture a target at the bifurcation of the middle cerebral artery, but, in the presence of a coil, anchoring at the stent site may have become strong, causing a vascular displacement on traction and leading to hemorrhage. Therefore, when selecting the size, a diameter smaller than used for thrombectomy must be adopted. Another issue is the selection of a framing coil. An Axium prime 3D, which we used, is a relatively flexible coil with an extrorse shape; therefore, it was inappropriate for a wide-neck aneurysm in the present case.
If there had been a decision to combine a neck bridge stent for the protrusion of the third coil into the parent artery, a series of coil migration would not have occurred.

\section{Conclusion}

We encountered a patient in whom coil retrieval with a stent retriever was successful in the management of coil migration. To our knowledge, no study has reported this procedure in Japan. However, it is technically simple and effective; this method should be considered in patients in whom coil retrieval with a microsnare or other devices is difficult.

\section{Disclosure Statement}

There is no conflict of interest for the main author and coauthors.

\section{References}

1) Wiebers DO, Whisnant JP, Huston J, et al: Unruptured intracranial aneurysms: natural history, clinical outcome, and risks of surgical and endovascular treatment. Lancet 2003; 362: 103-110.

2) Park HK, Horowitz M, Jungreis C, et al: Periprocedural morbidity and mortality associated with endovascular treatment of intracranial aneurysms. AJNR Am J Neuroradiol 2005; 26: 506-514.

3) Henkes H, Fischer S, Weber W, et al: Endovascular coil occlusion of 1811 intracranial aneurysms: early angiographic and clinical results. Neurosurgery 2004; 54: 268-280; discussion 280-285.

4) Vora N, Thomas A, Germanwala A, et al: Retrieval of a displaced detachable coil and intracranial stent with an L5 Merci Retriever during endovascular embolization of an intracranial aneurysm. $J$ Neuroimaging 2008; 18 : 81-84.

5) Ding D, Liu KC: Management strategies for intraprocedural coil migration during endovascular treatment of intracranial aneurysms. J Neurointerv Surg 2014; 6: 428-431.

6) Oh J, Kim J, Hong S, et al: Retrieval of unintended migrated detached coil: case report. J Cerebrovasc Endovasc Neurosurg 2014; 16: 268-274.

7) Liu KC, Ding D, Starke RM, et al: Intraprocedural retrieval of migrated coils during endovascular aneurysm treatment with the Trevo Stentriever device. J Clin Neurosci 2014; 21: 503-506.

8) Shigematsu T, Fujinaka T, Yoshimine T, et al: Endovascular therapy for asymptomatic unruptured intracranial 
aneurysms: JR-NET and JR-NET2 findings. Stroke 2013; 44: 2735-2742.

9) Roy D, Milot G, Raymond J: Endovascular treatment of unruptured aneurysms. Stroke 2001; 32: 1998-2004.

10) Pelz DM, Lownie SP, Fox AJ: Thromboembolic events associated with the treatment of cerebral aneurysms with Guglielmi detachable coils. AJNR Am J Neuroradiol 1998; 19: 1541-1547.

11) Casasco AE, Aymard A, Gobin YP, et al: Selective endovascular treatment of 71 intracranial aneurysms with platinum coils. J Neurosurg 1993; 79: 3-10.

12) Henkes $H$, Lowens $S$, Preiss $H$, et al: A new device for endovascular coil retrieval from intracranial vessels: alligator retrieval device. AJNR Am J Neuroradiol 2006; 27: 327-329.

13) $\mathrm{He} C$, Chen J, Hussain M, et al: Retrieval of a migrated coil with a handmade microwire-snare device. Acta Neurochir (Wien) 2016; 158: 1539-1543.
14) Schütz A, Solymosi L, Vince GH, et al: Proximal stent fixation of fractured coils: technical note. Neuroradiology 2005; 47: 874-878.

15) Chen Z, Tang W, Feng H, et al: Surgical extraction of migrated coils via proximal segment of the anterior cerebral artery: an emergency alternative. Neurol India 2009; 57: 327-330.

16) Leslie-Mazwi TM, Heddier M, Nordmeyer H, et al: Stent retriever use for retrieval of displaced microcoils: a consecutive case series. AJNR Am J Neuroradiol 2013; 34: 1996-1999.

17) Nikoubashman $O$, Pjontek R, Brockmann MA, et al: Retrieval of migrated coils with stent retrievers: an animal study. AJNR Am J Neuroradiol 2015; 36: 1162-1166.

18) O'Hare AM, Rogopoulos AM, Stracke PC, et al: Retrieval of displaced coil using a Solitaire(®) stent. Clin Neuroradiol 2010; 20: 251-254. 